Please do not remove this page

RMIT

UNIVERSITY

\title{
Reduction in urinary organophosphate pesticide metabolites in adults after a week-long organic diet
}

Oates, Liza; Cohen, Marc; Braun, Lesley; Schembri, Adrian; Taskova, Rilka

https://researchrepository.rmit.edu.au/esploro/outputs/9921859676901341/filesAndLinks?institution=61RMIT_INST\&index=null

Oates, L., Cohen, M., Braun, L., Schembri, A., \& Taskova, R. (2014). Reduction in urinary organophosphate pesticide metabolites in adults after a week-long organic diet. Environmental Research, 132, 105-111. https://doi.org/10.1016/j.envres.2014.03.021

Document Version: Accepted Manuscript

Published Version: https://doi.org/10.1016/j.envres.2014.03.021

Repository homepage: https://researchrepository.rmit.edu.au

(C) 2014 Elsevier Inc. All rights reserved.

Downloaded On 2023/04/27 01:30:35 +1000

Please do not remove this page 
Thank you for downloading this document from the RMIT Research Repository.

The RMIT Research Repository is an open access database showcasing the research outputs of RMIT University researchers.

RMIT Research Repository: http://researchbank.rmit.edu.au/

\section{Citation:}

Oates, L, Cohen, M, Braun, L, Schembri, A and Taskova, R 2014, 'Reduction in urinary organophosphate pesticide metabolites in adults after a week-long organic diet', Environmental Research, vol. 132, pp. 105-111.

See this record in the RMIT Research Repository at:

http://researchbank.rmit.edu.au/view/rmit:24443

Version: Accepted Manuscript

Copyright Statement: (c) 2014 Elsevier Inc. All rights reserved.

Link to Published Version:

http://dx.doi.org/10.1016/j.envres.2014.03.021 
NOTICE: this is the author's version of a work that was accepted for publication in Environmental Research. Changes resulting from the publishing process, such as peer review, editing, corrections, structural formatting, and other quality control mechanisms may not be reflected in this document. Changes may have been made to this work since it was submitted for publication. A definitive version was subsequently published in Environmental Research, Volume 132, July 2014, http://dx.doi.org/10.1016/j.envres.2014.03.021 


\title{
Accepted Author Manuscript
}

Final published version published online by Science Direct:

http://www.sciencedirect.com/science/article/pii/S001393511400067X

Oates, L., Cohen, M., Braun, L., Schembri, A., \& Taskova, R. (2014). Reduction in urinary organophosphate pesticide metabolites in adults after a week-long organic diet. Environmental Research, 132(0), 105-111. doi: http://dx.doi.org/10.1016/i.envres.2014.03.021

\section{Reduction in urinary organophosphate pesticide metabolites in adults after a week-long organic diet}

\author{
Liza Oates ${ }^{\mathrm{a}, *}$ \\ liza.oates@rmit.edu.au \\ Marc Cohen ${ }^{\mathrm{a}}$ \\ marc.cohen@rmit.edu.au \\ Lesley Braun ${ }^{\mathrm{b}, 1}$ \\ Adrian Schembric \\ Rilka Taskova $^{\mathrm{d}}$
}

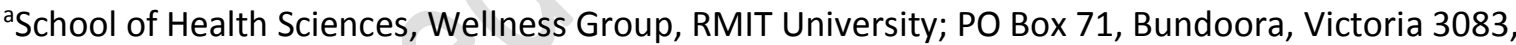
Australia

${ }^{b}$ Centre of Ethics in Medicine and Society, Department of Medicine, Monash University; Pharmacy Department, The Alfred Hospital, Melbourne, Australia

${ }^{c}$ CogState Limited, Melbourne, Australia

${ }^{\mathrm{d} A s u r e Q u a l i t y}$ Laboratories, Wellington, New Zealand

*Corresponding author.

${ }^{1} \mathrm{~L}$. Braun has moved since this work was completed and is now the Director of the Blackmore's Institute.

The research was reviewed and approved, prior to its conduct, by the Human Research Ethics Committee at RMIT University for the protection of human subjects (Ref.: 59/11). This work was supported in part by a donation to RMIT University from Bharat Mitra, co-founder of Organic India Pty Ltd. A follow-up study has received the financial support from Australian Organic. The individuals and organisations involved do not have any input into the studies and the authors' freedom to 
design, conduct, interpret and publish the research was not compromised. The authors declare they have no other actual or potential competing financial interests.

\section{Abstract}

Background Conventional food production commonly uses organophosphate (OP) pesticides, which can have negative health effects, while organic food is deemed healthier because it is produced without these pesticides. Studies suggest that organic food consumption may significantly reduce OP pesticide exposure in children who have relatively higher pesticide exposure than adults due to their different diets, body weight, behaviour and less efficient metabolism.

Objectives A prospective, randomised, crossover study was conducted to determine if an organic food diet reduces organophosphate exposure in adults.

Methods Thirteen participants were randomly allocated to consume a diet of at least $80 \%$ organic or conventional food for 7 days and then crossed over to the alternate diet. Urinary levels of six dialkylphosphate metabolites were analysed in first-morning voids collected on day 8 of each phase using GC-MS/MS with detection limits of0.11-0.51 $\mu \mathrm{g} / \mathrm{L}$.

Results The mean total DAP results in the organic phase were $89 \%$ lower than in the conventional phase $(\mathrm{M}=0.032[\mathrm{SD}=0.038]$ and $0.294[\mathrm{SD}=0.435]$ respectively, $\mathrm{p}=0.013)$. For total dimethyl DAPs there was a $96 \%$ reduction $(M=0.011[S D=0.023]$ and $0.252[S D=0.403]$ respectively, $p=0.005)$. Mean total diethyl DAP levels in the organic phase were half those of the conventional phase $(M=0.021$ $[S D=0.020]$ and $0.042[S D=0.038]$ respectively), yet the wide variability and small sample size meant the difference was not statistically significant.

Conclusions The consumption of an organic diet for one week significantly reduced OP pesticide exposure in adults. Larger scale studies in different populations are required to confirm these findings and investigate their clinical relevance.

Keywords: Biomonitoring; Organophosphate pesticides; Dialkylphosphate metabolites; Organic diets; Organic food

Abbreviations: DAP, dialkylphosphate; DEP, diethylphosphate;DETP, diethylthiophosphate; DEDTP, diethyldithiophosphate;DMP, dimethylphosphate; DMTP, dimethylthiophosphate;DMDTP, dimethyldithiophosphate; $\Sigma D A P$, total DAPs; $\Sigma E P$, total diethyl DAPs; $\Sigma M P$, total dimethyl DAPs; GC$\mathrm{MS} / \mathrm{MS}$, gas chromatography tandem mass spectrometry; LOD, limit of detection; LOQ, limit of quantification; ND, not detectable; NQ, not quantifiable; OC, organochlorine pesticides; OFIS, Organic Food Intake Survey; OP, organophosphate pesticide

\section{Introduction}

Organophosphate pesticides (OPs) are widely used in conventional agriculture (Radcliffe, 2002) and have replaced many organochlorines (OCs) such as DDT and dieldrin, which are lipophilic and bioaccumulatic. While OPs are not as persistent as OCs, they are more acutely toxic and act to irreversibly inhibit acetylcholinesterase, an enzyme critical to nerve function in both insects and humans. The neurotoxic effects of OPs are not specific to their target species and at high doses OPs are fatal, while at sublethal doses OPs cause a predictable cholinergic syndrome that can progress to paralysis and respiratory arrest. OPs are absorbed by all routes, including ingestion, inhalation, and dermal absorption and humans are commonly exposed to OPs via ingested food and drink and inhalation of contaminated air, as well as being exposed through occupational contact and proximity 
to farms (Babina et al., 2012; Bouvier et al., 2005; Fenske et al., 2000). As OPs are mostly metabolised in the liver, susceptibility to the harmful effects from OPs may vary with genetic, developmental or environmentally-induced differences in detoxification enzyme activity (Buratti et al., 2007; Holland et al., 2006).

OP residues are frequently detected in foods at low levels in Australia and elsewhere (FSANZ, 2011; The Expert Committee on Pesticide Residues in Food, 2013; United States Department of Agriculture, 2013) and a number of human biomonitoring studies have assessed OP exposure through measures of urinary dialkylphosphates (DAPs), which are non-selective OP metabolites representing 70-80\% of the OP class (Babina et al., 2012; Barr et al., 2004; Bouvier et al., 2005; Health Canada, 2011; Heudorf et al., 2004). In a study of 48 non-occupationally exposed Australian adults, one or more DAPs were detected in all samples (Oglobline et al., 2001) and a recent study of South Australian preschool children found widespread chronic exposure to multiple OPs at levels higher than similar populations in the US or Germany (Babina et al., 2012).

There is currently little data on the health effects of chronic, low-dose OP exposure in the general population. In occupational settings, chronic low-dose exposure has been shown to impair neurobehavioual function (Ross et al., 2013) and prenatal OP exposure has been associated with shorter gestational age and reduced birth-weight along with poorer intellectual development and higher prevalence of ADHD in children (Bouchard et al., 2010, 2011; Rauch et al., 2012). While there is an increasing amount of biomonitoring data reporting the extent of pesticide exposure in the general population, there are few reports of interventions that may reduce this exposure.

The main route of OP exposure for the general population appears to be through the diet (Lu et al., 2008; Morgan et al., 2005; Wilson et al., 2003) and many consumers are turning to organic food in an attempt to reduce their pesticide exposure (Oates et al., 2012). However, humans are also exposed to non-dietary pesticides through inhalation of contaminated air, absorption through the skin or accidental ingestion. As such the extent to which an organic diet modifies overall exposure is unclear. Nevertheless, organic food sales are increasing in Australia (Monk et al., 2012) and elsewhere (Willer et al., 2013) and this growth is fuelled by the belief that organic food is healthier than conventionally grown food because it contains fewer pesticide residues (Oates et al., 2012). This belief is supported by studies in children that have demonstrated dramatic reductions in OP exposure with the consumption of organic food (Curl et al., 2003; Lu et al., 2006, 2008). It is unclear however, if the results of these studies can be extrapolated to adults, as children have higher pesticide exposure levels than adults due to their different diets, body weights, and behaviours (Landrigan and Garg, 2005) and reduced activity of enzymes required for their metabolism (Huen et al., 2009). To date no published studies have examined the effects of organic food consumption on urinary OP metabolites in adults.

The purpose of this study was to determine if consumption of a mostly organic diet leads to significantly reduced urinary organophosphate pesticide metabolites in adults.

\section{Materials and methods}

\subsection{Study design}

A prospective, randomised, single-blinded, crossover study was conducted to compare the levels of DAP metabolites in the urine of adults after a week of consuming mostly conventional foods and a week consuming mostly organic foods. The study was conducted in Melbourne, Australia, between December 2011 and November 2012 and approved by the RMIT University Human Research Ethics Committee. 


\subsection{Study population}

Subjects were recruited through the distribution of postcards available at retail outlets and through a variety of electronic sources including social media sites such as Facebook and Twitter. Links to a website provided information about the purpose and conduct of the study. Prospective participants were screened by telephone to confirm their eligibility before being sent the participant information and consent forms. No incentives, financial or otherwise, were offered to participants other than an offer to receive their test results at the end of the study period.

\subsection{Inclusion}

Participants were non-smoking adults aged between 18 and 65 living in urban or periurban areas and able to provide consent and comply with the study protocol, which included consuming a mostly (at least $80 \%$ ) organic or conventional diet and completion of study documents and online surveys.

\subsection{Exclusion}

Exclusion criteria were chosen to reduce the effect of possible confounders and included the following:

- Use of tobacco products as tobacco use has been positively correlated with urinary pesticide levels (Riederer et al., 2008).

- Medical conditions or medication use that may interfere with the absorption, metabolism or elimination of pesticides.

- Pregnancy or lactation, as the expression of enzymes that detoxify pesticides may be down regulated during pregnancy (Fortin et al., 2012) and there was a potential for psychological distress if abnormally high pesticide results were identified in pregnant or lactating women.

- Being unable or unwilling to comply with the study protocol.

\subsection{Group allocation and blinding}

The order in which subjects undertook the organic and conventional phases of the study was randomly assigned by block randomisation (block size of 4) using StatsDirect Statistical Software (https://statsdirect.com). Double-blinding was not possible as participants knew which diet they were eating, but all documents and specimen containers were coded so that laboratory technicians were blinded to subjects' identity and group allocation.

\subsection{Intervention}

Participants were asked to maintain their usual dietary choices and to source their own food, which could be obtained from a variety of locations including supermarkets, farmer's markets and local grocers. Participants were also asked to consume as close to $100 \%$ conventional or organic food as possible during each 7-day dietary period.

On day 8 of each period, participants provided a first morning urine sample and completed a short survey on factors that may affect chemical exposure. This included questions related to potential non-dietary sources of pesticide exposure, time spent in parks/gardens/rural areas, and food preparation techniques that may affect dietary pesticide exposure. Participants were then crossed over to the alternate diet for a 7-day period. A washout period was not required; however participants were permitted to take a break between the study phases if they desired. 


\subsection{Outcomes}

\subsubsection{Organic Food Intake Survey (OFIS)}

A detailed diet diary, known as the Organic Food Intake Survey (OFIS) was used to determine the amount of organic or conventional food consumed during each dietary phase as well as the number and category of servings. The OFIS used a modified version of the Australian Guide to Healthy Eating (AGHE) (Department of Health and Aging [DHA], 2008) food group categories and serving sizes. This allowed for quantification of organic versus conventional food intake by the number of servings from each food category. Participants were asked to record any organic produce consumed as either 'certified organic', 'likely organic', 'likely conventional' or 'unknown' in the OFIS worksheet. The OFIS, which had been previously tested in a pilot study was accompanied by detailed instructions and a sample worksheet and included pictures of the various certification logos used in Australia which adhere to 'The Standard' to aid participants in identifying certified organic food sources (AQIS, 2009). Once submitted, OFIS details were checked by a nutritionist and participants were contacted to resolve any queries.

Previous research had revealed that it was unrealistic to expect participants to consume a $100 \%$ organic diet during the organic phase (Oates et al., 2012). In order to ensure that the diets were sufficiently different between phases with respect to the amount of organic and conventional produce, there was an a priori requirement that participants consume at least $80 \%$ of their food servings from organic or conventional food during the relevant phase. Participants were not given this figure and were simply asked to 'consume as much organic/conventional food as they could' during the phase. The OFIS also provided a means of assessing whether the total number of food servings and servings from each food category was similar in each phase.

\subsubsection{Urine}

First morning void urine samples were used as they are suggested to provide the best representative measurement of daily OP pesticide exposure when using spot samples (Kissel et al., 2005). On the morning of day 8, participants provided a coded, $200 \mathrm{~mL}$ first morning void, spot urine sample. This was transported on ice to the Tullamarine branch of AsureQuality Laboratories where it was stored at $-18^{\circ} \mathrm{C}$ before being sent to the AsureQuality Wellington (NZ) laboratory where it remained stored frozen $\left(-18^{\circ} \mathrm{C}\right)$ or cold $\left(4^{\circ} \mathrm{C}\right)$ prior to analysis.

\subsection{Urinalysis}

Urinalysis of six DAP metabolites of OP pesticides, DMP, DMTP, DMDTP, DEP, DETP and DEDTP, was performed using gas chromatography tandem mass spectrometry (GC-MS/MS) by AsureQuality Limited, which is accredited to NZS ISO/IEC 17025:2005 by International Accreditation New Zealand (IANZ:131).

The extraction method was based on Bravo et al. (2004) and utilises an extra purification step that reduces analytical interferences and affords lower detection limits. Briefly, $5 \mathrm{~mL}$ of urine was mixed with $3 \mathrm{~mL}$ of ion-pairing reagent (100 mM tetrabutylammonium hydrogen sulphate) and purified by reversed phase C18 solid phase extraction. Dialkylphosphate fraction was eluted with acetonitrile and concentrated to dryness. The residue was reconstituted in acetonitrile and DAPs derivatised with 1-chloro-3-iodopropane to chloropropyl esters. The latter were analysed by GC-MS/MS on an Agilent 7890A GC system coupled with an Agilent 7000 GC/MS Triple Quad with an El source (Agilent Technologies, Santa Clara, CA, USA). 
Results were reported to two significant figures in micrograms per litre $(\mu \mathrm{g} / \mathrm{L})$ present in the sample on an 'as received' basis. Additionally, values corrected for the level of creatinine were reported to two significant figures in micrograms per gram of creatinine $(\mu \mathrm{g} / \mathrm{g}$ creatinine).

\subsection{Quality control/quality assurance}

The method was validated and accredited to ISO17025 standard. The validation set consisted of five batches of recovery samples; linearity test comprising fortification at nine levels; estimates of repeatability, intermediate precision and uncertainty of measurement were based on 26 fortifications at three different levels. The limits of detection (LODs) were determined as $0.42 \mathrm{\mu g} / \mathrm{L}$ for DMP, $0.51 \mu \mathrm{g} / \mathrm{L}$ for DEP, $0.28 \mu \mathrm{g} / \mathrm{L}$ for DMTP, $0.16 \mu \mathrm{g} / \mathrm{L}$ for DETP, $0.14 \mu \mathrm{g} / \mathrm{L}$ for DMDTP and 0.11 $\mu \mathrm{g} / \mathrm{L}$ for DEDTP. A reagent blank test, a matrix blank test, at least three fortified blank samples and a spiked QC sample were analysed with each batch of unknown samples.

\subsection{Quantification limits and creatinine correction}

Creatinine was used as a marker for urine dilution and DAP concentration was adjusted by the creatinine concentration in the sample. Urinary creatinine concentration was determined by an automated colorimetric method on a Roche Modular P800 clinical analyser (Roche Diagnostics, Indianapolis, USA) at Aotea Patology, Wellington, New Zealand.

\subsection{Data analysis}

Data analysis was performed using SPSS for Windows statistical software (version 18). For the purpose of determining central tendency and dispersion (variability) numerical figures are required, so assumptions were made to deal with non-detectable (ND) and non-quantifiable (NQ) results. As with previous studies (Curl et al., 2003; Oglobline et al., 2001) all samples containing concentrations below the limits of detection were assumed to have a concentration equal to one half of the LOD. Samples containing concentrations below the LOQ were assumed to have a concentration equal to the midpoint between the LOD and the LOQ. These are referred to as the adjusted results and were used to calculate measures of central tendency and dispersion (variability). The detection and quantification limits including the adjusted ND and NQ of the six DAPs measured are presented in Table 1.

Table 1. LOD, LOQ, adjusted ND and NQ of the DAPs

\begin{tabular}{lcccc}
\hline DAP & LOD $^{\mathrm{a}}$ & LOQ $^{\mathrm{b}}$ & Adjusted ND $^{\mathrm{c}}$ & Adjusted NQ $^{\mathrm{d}}$ \\
\hline Dimethylphosphate (DMP) & 0.42 & 1.3 & 0.210 & 0.860 \\
Diethylphosphate (DEP) & 0.51 & 1.5 & 0.255 & 1.005 \\
Dimethylthiophosphate (DMTP) & 0.28 & 0.83 & 0.140 & 0.555 \\
Diethylthiophosphate (DETP) & 0.16 & 0.49 & 0.080 & 0.325 \\
Dimethyldithiophosphate (DMDTP) & 0.14 & 0.43 & 0.070 & 0.285 \\
Diethyldithiophosphate (DEDTP) & 0.11 & 0.33 & 0.055 & 0.220 \\
\hline
\end{tabular}

${ }^{a}$ Limits of detection 
${ }^{b}$ Limits of quantification

${ }^{c}$ Not detected, levels below the LOD

${ }^{d}$ Not quantified, levels greater than or equal to the LOD and less than the LOQ

In line with a previous study of DAPs in children consuming organic or conventional food (Curl et al., 2003), we calculated the molar sums of the dimethyl-containing and diethyl-containing metabolites so that each participant received a score for combined total DAPs (LDAP), total dimethyl DAPs (LMP) and total diethyl DAPs ( $\Sigma E P)$. To calculate the total molar metabolite quantities $(\mu \mathrm{mol} / \mathrm{g})$ for each participant, the individual DAP result $(\mu \mathrm{g} / \mathrm{g})$ was divided by its molecular weight $(\mathrm{g} / \mathrm{mol})$ before being added together.

$\sum \mathrm{MP}=[\mathrm{DMP}] / 125+[\mathrm{DMTP}] / 141+[\mathrm{DMDTP}] / 157$

$\sum \mathrm{EP}=[\mathrm{DEP}] / 153+[\mathrm{DETP}] / 169$

$\Sigma D A P=\Sigma M P+\Sigma E P$

\section{Results}

\subsection{Study participants}

A total of 13 matched samples were available for the group analysis. Fifteen participants living in the greater Melbourne area of Victoria were enrolled with one withdrawing for personal reasons after completing the first phase. Results from a further participant's data were excluded from the group analysis as the participant did not consume at least $80 \%$ organic food during that phase. Over the week this participant consumed an average of $65 \%$ organic servings during the organic phase with consumption being below $50 \%$ organic on a number of days. The demographic characteristics of the included participants are presented in Table 2.

Table 2. Demographics $(N=13)$ of BMT Participants

\section{$n(\%)$}

\section{Gender}

Female

Male

\section{Location}

Urban

Periurban

$9(69)$ 
Participants were mostly female living in urban and periurban (suburban or semi-rural with no nearby agriculture) areas with a mean age of 42.1 years ( $S D=10.15$ years). On average, $96 \%$ of their food servings were from conventional produce during the conventional phase and $93 \%$ from organic produce in the organic phase (this included $83 \%$ certified organic). The mean total number of food servings and servings from each food category was very similar in each phase (Table 3 ) with the exception that participants consumed significantly less animal protein during the organic phase compared to the conventional phase $(-36 \%, p=0.006)$.

Table 3. Average Number of Daily Servings by Food Category

\begin{tabular}{lcccc}
\hline Food category & $\begin{array}{c}\text { Conventional phase } \\
\text { (Mean) }\end{array}$ & $\begin{array}{c}\text { Organic phase } \\
\text { (Mean) }\end{array}$ & $\begin{array}{c}\text { Difference } \\
\text { Organic vs. }\end{array}$ & Sig \\
Conventional & & \\
\hline Grain & 2.5 & 2.6 & $+6 \%$ & .456 \\
Vegetables & 3.6 & 3.9 & $+8 \%$ & .272 \\
Fruit & 3.1 & 3.1 & $+1 \%$ & .917 \\
Dairy & 1.5 & 1.4 & $-9 \%$ & .328 \\
Animal protein & 1.3 & 0.9 & $-36 \%$ & $.006 *$ \\
Vegetable protein & 1.0 & 1.2 & $+24 \%$ & .136 \\
Total food servings & 13.0 & 13.2 & $\sim+2 \%$ & .807 \\
\hline
\end{tabular}

a Difference in the average number of food category servings in the organic compared to the conventional phase

${ }^{*} p<.05$ (Wilcoxon Signed-Ranks Test)

\subsection{Statistical considerations}

Total molar metabolite quantities ( $\mu \mathrm{mol} / \mathrm{g}$ ) were calculated for each participant. DEDTP was not included in any calculations due to the very low frequency of quantifiable detections of this metabolite, which is in line with the previous study by Curl et al. (2003). As the change in DAP scores did not appear normally distributed and the sample size was not large enough to apply the central limit theorem, non-parametric statistical tests were used to test for a statistically significant change in DAP scores between the conventional and organic diets (Field, 2013). The Wilcoxon signed-ranks test was used, which is a non-parametric alternative to the paired sample t-test that does not require the assumption of normally distributed change scores. The use of non-parametric tests is consistent with the previous study (Curl et al., 2003). The dose estimation methods used, choice of non-parametric tests for statistical analysis and use of creatinine correction do not appear to have influenced the direction of the findings. 


\subsection{Rates of detection}

At least one metabolite was detected at quantifiable levels in all of the 13 participants during the conventional phase (Table 4). In the organic phase four of the 13 participants (31\%) had no quantifiable detections of which two (15\%) had no detectable levels of DAP metabolites. The most frequently detected metabolites were DMTP and DEP in the conventional phase and DEP in the organic phase. DMTP was detected at quantifiable levels in all but one of the conventional samples and in five of the organic samples, although only two were at quantifiable levels. DEP was the most commonly detected metabolite in the organic phase with 10 detections of which six were at levels that could be quantified.

Table 4. Frequency of Detection for Individual Metabolites.

Frequency of detection ${ }^{\mathrm{a}}$

Frequency of quantifiable detections

\begin{tabular}{lcccccc}
\hline & $\begin{array}{c}\text { Overall } \\
n=26 \\
(\%)\end{array}$ & Con $n=13(\%)$ & $\begin{array}{c}\text { Org } n=13 \\
(\%)\end{array}$ & $\begin{array}{c}\text { Overall } \\
n=26(\%)\end{array}$ & $\begin{array}{c}\text { Con } n=13 \\
(\%)\end{array}$ & Org $n=13(\%)$ \\
DMP & $6(23)$ & $6(46)$ & $0(0)$ & $5(19)$ & $5(38)$ & $0(0)$ \\
DEP & $21(81)$ & $11(85)$ & $10(77)$ & $14(54)$ & $8(62)$ & $6(46)$ \\
DMTP & $17(65)$ & $12(92)$ & $5(38)$ & $14(54)$ & $12(92)$ & $2(15)$ \\
DETP & $14(54)$ & $8(62)$ & $6(46)$ & $12(46)$ & $7(54)$ & $5(38)$ \\
DMDTP & $9(35)$ & $8(62)$ & $1(8)$ & $8(31)$ & $7(54)$ & $1(8)$ \\
DEDTP & $4(15)$ & $3(23)$ & $1(8)$ & $3(12)$ & $3(23)$ & $0(0)$ \\
\hline
\end{tabular}

${ }^{\mathrm{a}}$ Includes both quantifiable and non-quantifiable detections (>LOD).

\subsection{Individual DAPs}

Differences in mean urinary DMP and DMTP levels were statistically significant between the conventional and organic phases $(p<0.05)$ and there was a trend towards significance for differences in DMDTP (Table 5).

Table 5. DAP Results for Individual Metabolites (Creatinine Corrected $\mu \mathrm{g} / \mathrm{g}$ ) N=13

\begin{tabular}{lccccc} 
Metabolite & \multicolumn{2}{c}{ Maximum } & & Mean (Standard Deviation) & Sig $^{\mathrm{a}}$ \\
& \multicolumn{2}{c}{$\mu \mathrm{g} / \mathrm{g}$} & \multicolumn{2}{c}{$\mu \mathrm{g} / \mathrm{g}$} \\
& $\mathrm{Con}^{\mathrm{b}}$ & $\mathrm{Org}^{\mathrm{c}}$ & $\operatorname{Con}^{\mathrm{b}}$ & $\operatorname{Org}^{\mathrm{c}}$ & \\
\hline DMP & 23 & $\mathrm{ND}^{\mathrm{d}}(0.21)^{\mathrm{e}}$ & $3.9(6.7)$ & $\mathrm{ND}^{\mathrm{d}}(-)$ & $.028^{*}$ \\
\hline
\end{tabular}




\begin{tabular}{lccccc}
\hline DEP & 12 & 7.4 & $4.8(4.5)$ & $2.8(2.6)$ & .221 \\
DMTP & 160 & 8.5 & $29(48)$ & $0.98(2.3)$ & $.005^{*}$ \\
DETP & 10 & 3.6 & $1.8(3.4)$ & $0.56(0.97)$ & .263 \\
DMDTP & 14 & 3.7 & $2.3(3.9)$ & $0.35(1.0)$ & $.051^{* *}$ \\
DEDTP & 0.33 & $\mathrm{NQ}^{f}(0.22)^{\mathrm{e}}$ & $0.12(1.2)$ & 0.068 & .144 \\
& & & & $(0.046)$ & \\
\hline
\end{tabular}

Note: Results reported to two significant figures. Minimums not presented as all were ND

a Significance of the difference between the conventional and organic phase

${ }^{b}$ Conventional phase

c Organic phase

${ }^{d}$ Not detectable, levels below the LOD

e This value was used because there were no values that were detectable/ quantifiable

${ }^{f} \mathrm{NQ}=$ Not quantifiable, levels greater than or equal to the LOD but less than the LOQ

${ }^{*} p<.05$ (Wilcoxon Signed-Ranks Test)

${ }^{* *}$ Trend towards significance

Fig. 1 presents a comparison of the mean creatinine corrected results for each of the dimethyl metabolites (DMP, DMTP, and DMDTP). In the conventional phase there was a considerable amount of variability in DMTP results. In the organic phase only two samples had quantifiable detections for DMTP and one had a quantifiable level of DMDTP with DMP returning no quantifiable results (Fig. 1). 


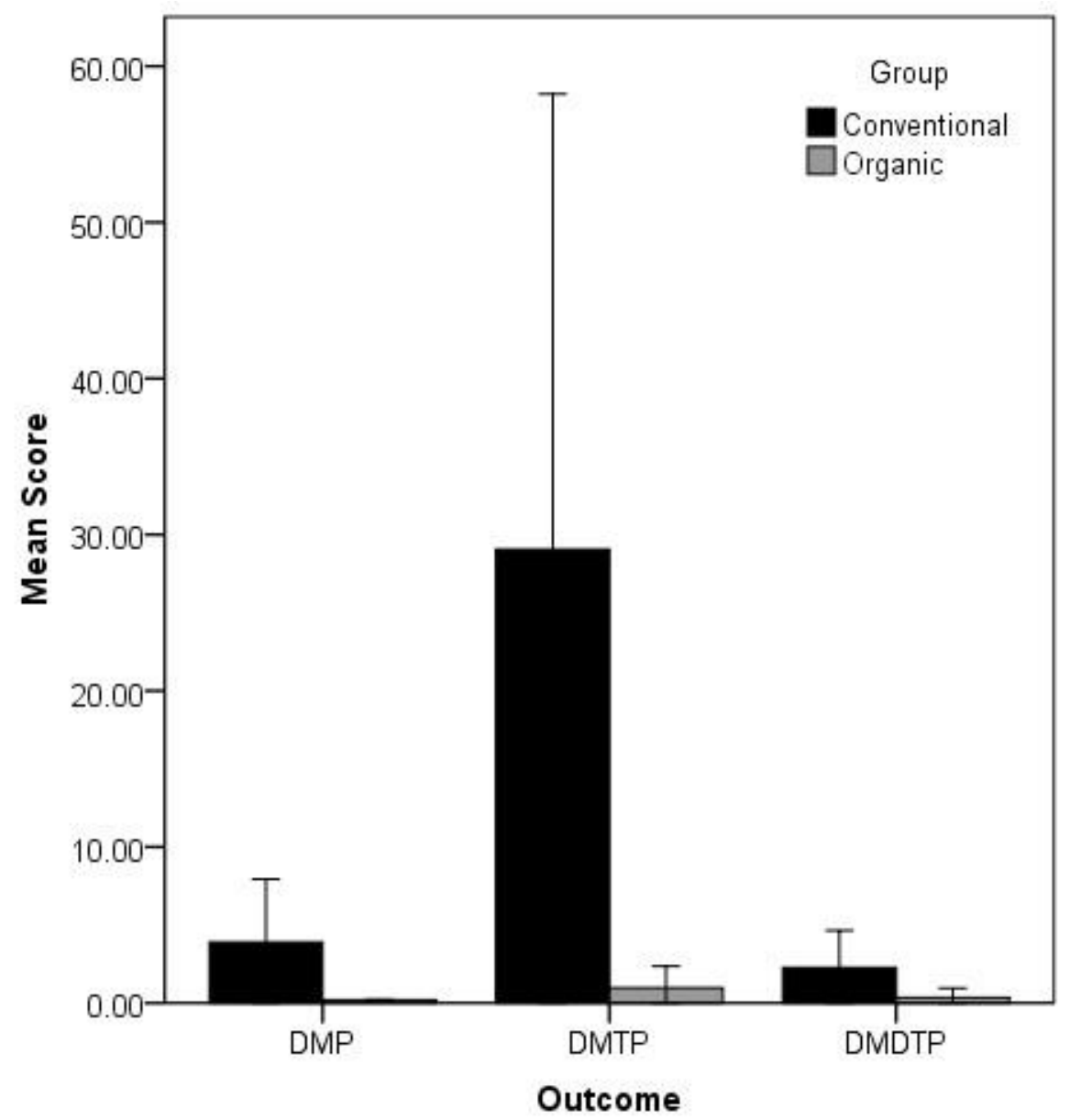

Fig. 1 Mean dimethyl DAPs (creatinine corrected) $\mathrm{N}=13$.

Compared to the dimethyl DAPs (DMP, DMTP, and DMDTP) (Fig. 1), the diethyl DAP results (DEP, DETP, and DEDTP) (Fig. 2) were more variable and there were no statistically significant differences between dietary phases (Table 4). 


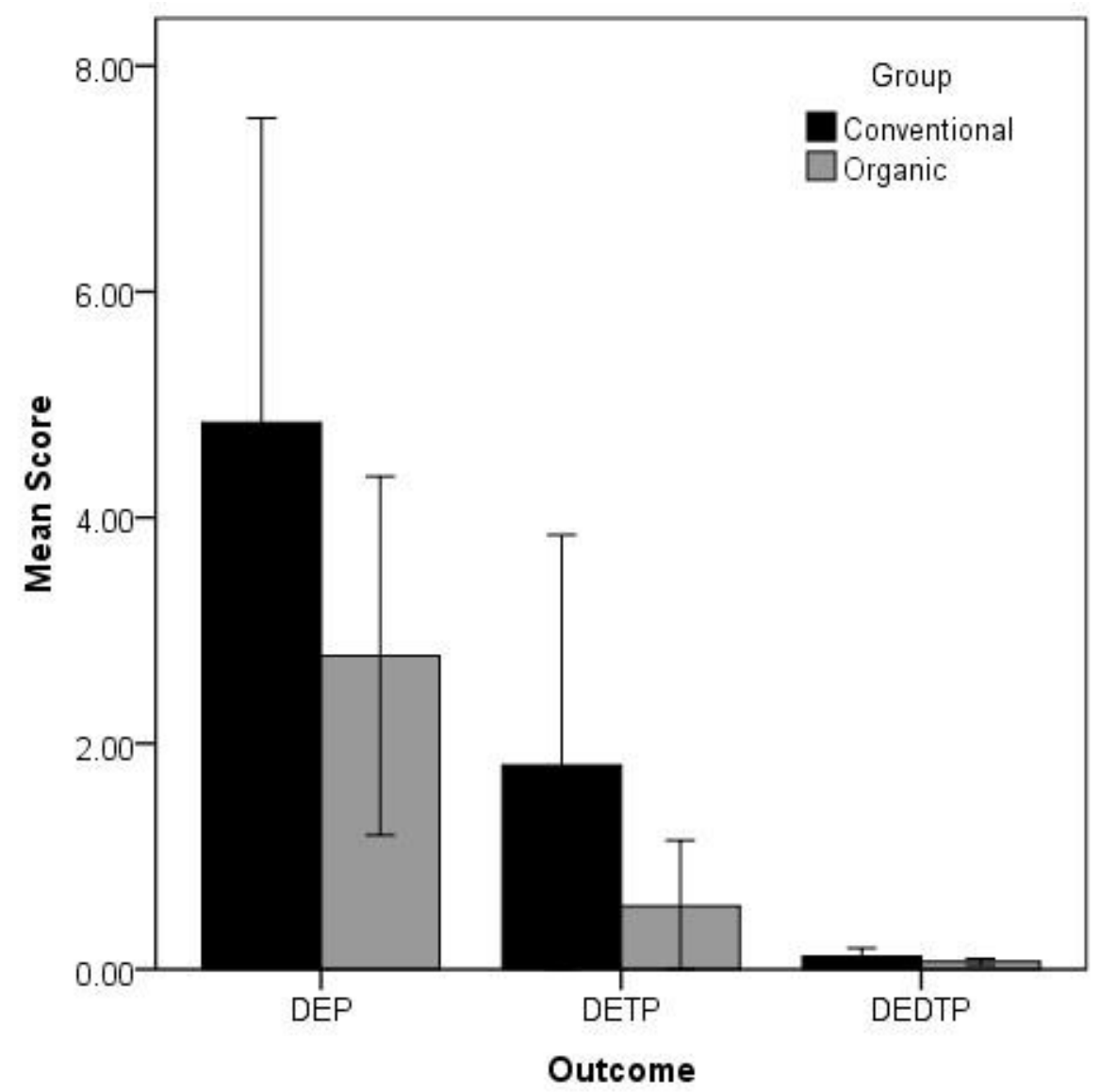

Fig. 2 Mean diethyl DAPs (creatinine corrected). $\angle L O Q=$ below the limits of quantification.

\subsection{Total DAP results ( $($ DAP)}

Both $\Sigma D A P$ and $\Sigma M P$ were significantly higher in the conventional phase than the organic phase (Fig. 3). The mean $\Sigma D A P$ results in the organic phase were $89 \%$ lower than in the conventional phase $(M=0.032 \pm 0.038$ and $0.294 \pm 0.435$ respectively, $p=0.013)$. For $\Sigma M P$ there was a $96 \%$ reduction $(M=0.011 \pm 0.023$ and $0.252 \pm 0.403$ respectively, $p=0.005)$. Although the mean $\Sigma E P$ levels were reduced by $49 \%$ in the organic compared to the conventional phase $(M=0.021 \pm 0.020$ and $0.042 \pm 0.038$ respectively, $\mathrm{p}=0.170$ ) this difference was not statistically significant. 


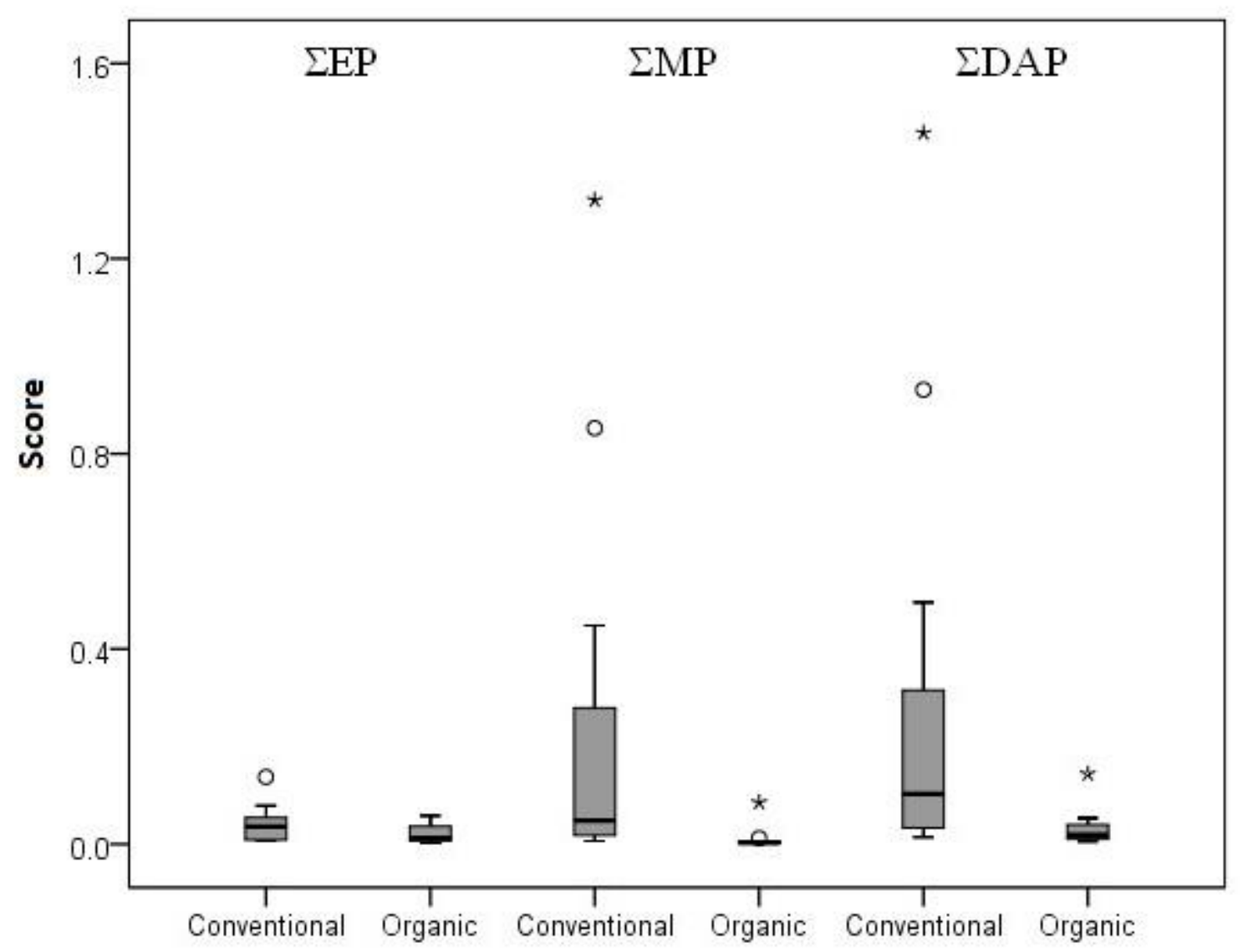

Fig. 3 $\Sigma$ DAP, $\Sigma \mathrm{MP}$ and $\Sigma$ EP (creatinine corrected). Mild outliers are marked with a circle (O) and extreme outliers are marked with an asterisk $(*)$ on the boxplot.

\section{Discussion}

The finding of reduced levels of urinary DAPs in adults after a week of eating mostly organic food suggests that there is greater dietary exposure to OP pesticides from conventionally produced foods than organic foods and that consumption of organic food significantly reduces this exposure. The reduction of total DAP levels by nearly $90 \%$ after a week, further suggests that the vast majority of ingested OP pesticides are metabolised and excreted within a week of ingestion. These findings support previous studies on children that demonstrate a reduction of OPs with consumption of organic food (Curl et al., 2003; Lu et al., 2006).

\subsection{Factors influencing pesticide exposure}

Although pesticide metabolite levels were dramatically lower in the organic phase, they were still detected in some samples. There are a number of possible reasons for this, the most likely being exposure to non-dietary sources of OPs, which may be used in homes, gardens, parks and public land and can be inhaled or absorbed through the skin.

While it is not possible to attribute the levels of individual DAPs to specific pesticides, non-dietary exposure may be particularly relevant for diethyl DAPs, which did not show any statistically significant differences across the phases. This is consistent with results reported by Curl et al. (2003) and is supported by the finding of more than 10 times greater urinary diethyl DAP concentrations in children living in periurban or rural areas than those in urban dwellings (Babina et al., 2012). This 
suggests that agricultural pesticide use in rural and periurban areas may increase non-dietary exposure to pesticides that metabolise to DEP (CDC, 2008). Diethyl DAPs may also have been present due to contamination of organic produce. Although rare, this has been previously reported to occur during production, transport or storage both in Australia (McGowan, 2003) and abroad (Baker et al., 2002; Tasiopoulou et al., 2007; USDA, 2012). It is also possible that the small amounts of conventional food eaten during the organic phase had high levels of contamination from pesticides that favour the production of diethyl DAPs.

\subsection{Comparison to other populations.}

The mean DAP metabolites in the conventional phase of our study ( 0.12 for DEDTP to 29 for DMTP, refer to Table 5 in results) are comparable to the only Australian study of non-occupationally exposed adults ( $n=48$ ) (Oglobline et al., 2001), yet are substantially lower than the four DAPs reported in urban dwelling children in South Australia (Babina et al., 2012). This may reflect that children are disproportionately exposed to pesticides due to their lower body weights and slower metabolism of pesticides. The detection rates for DAP metabolites from our study are also comparable to the results from the NHANES 1999-2000 adult subgroup (20-59 years; $n=814$ ) (Barr et al., 2004), yet our study reported lower rates of detection of DMTP, (92\% compared to $63 \%$ in the NHANES). This may indicate that pesticides that metabolise to DMTP may be used more in Australia and this is supported by high frequencies of detection for DMTP being reported in Australian studies of non-occupationally exposed adults (Oglobline et al., 2001) and South Australian children (Babina et al., 2012).

The only published study to compare DAP levels between organic and conventional consumers was conducted in children aged 2-5 years (Curl et al., 2003). The children consuming organic fruit, vegetables and juice $(n=18)$ had significantly lower levels of total dimethyl DAPs in their urine than children consuming conventional $(n=21)$ produce $(p=0.0003)$. Uncorrected results were used and the mean values differed by a factor of nine $(0.04$ and $0.34 \mu \mathrm{mol} / \mathrm{L})$. The total diethyl DAPs did not differ significantly across the two groups $(p=0.13)$. These findings are in line with the present study despite differences in metabolism between children and adults.

While it is suggested that excretion of DAP metabolites is usually quite rapid with $80-90 \%$ being excreted within $48 \mathrm{~h}$ (Aprea et al., 2002), there may be factors such as genetic polymorphisms in detoxification pathways, medication use and pathological processes that may compromise an individual's ability to metabolise and excrete DAPs. Currently there is insufficient information to be able to predict these effects, yet previous studies have found a rapid reduction in specific OP metabolites in 23 children upon commencement of an organic diet (Lu et al., 2006).

\subsection{Consumption patterns of participants}

Unlike previous studies that have compared different populations eating either mostly conventional or organic produce (Curl et al., 2003) or that specifically replaced conventional food items with organic food (Lu et al., 2006), our study involved the same individuals who were required to source and purchase their own conventional and organic food. This provides the study with external validity and relevance to the general community and allowed the study to be conducted with limited resources. Results from the OFIS suggest that participants' diets did not deviate widely across the two phases (Table 3 ) although there were more servings of dairy consumed in the conventional phase and more grain, vegetable and fruit servings consumed during the organic phase. The only statistically significant difference was that participants consumed more animal protein in the conventional phase, conversely there was more vegetable protein in the organic phase (nonsignificant), which may reflect the cost and availability of organic meat. 


\subsection{Limitations}

The study period ran over almost a full year cycle with different participants completing the study at different times. While participants provided their own 'within season' comparisons, the sample was too small to investigate any seasonal variation in exposure levels. Such seasonal variation may be expected however, as higher pesticide residues have been reported in spring and summer months (MacIntosh et al., 2001) and intake of fruit and vegetables is reported to be higher in the summer months (Lu et al., 2008). Participants may also have been more inclined to spend time outdoors in parks and rural areas which may have increased the risk of non-dietary exposure.

This study had a small sample size and while the reported differences and their significance values suggest a dramatic reduction in OP exposure is possible with an organic diet, the adults who participated in this study are not necessarily representative of the wider community and regional differences in pesticide use and food consumption mean that our results may not be applicable to different populations or regions. Furthermore, DAP metabolites are produced by over $70 \%$ of pesticides in the OP class and therefore the analysis of DAP metabolites makes it impossible to attribute elevated urinary DAPs to specific foods, behaviours or pesticides. There are also many confounding factors that determine risk associated with pesticide exposure for any individual, including age, comorbidities, concurrent medication use, the timing of exposure, interactions with other chemicals and genetic, developmental or environmentally induced differences in detoxification capacity. Therefore without further epidemiological and toxicological data it is impossible to determine a urinary DAP concentration that corresponds with negative health impacts in the general population (Sudakin and Stone, 2011).

While emerging research shows an association between adverse effects and chronic low dose dietary exposure to OPs during critical periods of development (Bouchard et al., 2010, 2011; United States Department of Agriculture, 2013), with a linear response between pre-natal OP exposure and subsequent cognitive impairment with no threshold effect (Bouchard et al., 2011), the clinical significance of reducing OP exposure with an organic diet is unclear. The finding of dramatic reductions in OP pesticide exposure with an organic diet however, suggests that consumption of organic food is a practical way to minimise exposure as part of a precautionary approach. Further large scale studies with clinical endpoints are now required to determine any associated health risks of OP exposure in adults and any associated health benefits of reducing pesticide exposure through the consumption of organic food.

\subsection{Conclusions}

Australian adults are exposed to OPs through conventional diets and this exposure can be dramatically reduced after a week consuming mostly organic food. Future large scale studies investigating the relationship between exposure and health outcomes are required to determine if the reduction in OPs associated with an organic diet has clinical relevance. Nevertheless, the consumption of organic food provides a logical precautionary approach to reducing pesticide exposure.

\section{Acknowledgments}

We thank J. Baglin for his assistance with data analysis, the team at AsureQuality Pty Ltd. (New Zealand) for measuring the urinary concentrations of dialkylphosphates, and the research subjects for their participation. 


\section{References}

Aprea C., et al., Biological monitoring of pesticide exposure: a review of analytical methods, J. Chromatogr. B: Anal. Technol. Biomed. Life Sci. 769, 2002, 191-219, DOI: 10.1016/S15700232(02)00044-2.

AQIS, National Standard for Organic and Bio-Dynamic Produce-Edition 3.4 July 2009. Australian Quarantine \& Inspection Service, 2009.

Babina K., et al.,Environmental exposure to organophosphorus and pyrethroid pesticides in South Australian preschool children: a cross sectional study, Environ. Int. 48, 2012, 109-120, DOI: 10.1016/j.envint.2012.07.007.

Baker B.P., et al., Pesticide residues in conventional, integrated pest management (IPM)-grown and organic foods: insights from three US data sets, Food Addit. Contam. 19, 2002, 427-446.

Barr D.B., et al., Concentrations of dialkyl phosphate metabolites of organophosphorus pesticides in the U.S. population, Environ. Health Perspect. 112, 2004, 186-200 〈http://www.ncbi.nlm.nih.gov/pmc/articles/PMC1241828/〉.

Bouchard M.F., et al., Attention-deficit/hyperactivity disorder and urinary metabolitesof organophosphate pesticides, Pediatrics 125, 2010, e1270-e1277, DOI: 10.1542/peds.2009-3058.

Bouchard M.F., et al., Prenatal exposure to organophosphate pesticides and IQ in 7-year old children, Environ. Health Perspect. 119, 2011, 1189-1195, DOI: 10.1289/ehp.1003185.

Bouvier G., et al., Insecticide urinary metabolites in nonoccupationally exposed populations, J. Toxicol. Environ. Health B: Crit.Rev. 8, 2005, 485-512, DOI: 10.1080/10937400591007284.

Bravo R., et al., Measurement of dialkyl phosphate metabolites of organophosphorus pesticides in human urine using lyophilization with gas chromatography-tandem mass spectrometry and isotope dilution quantification, J. Expo. Anal. Environ. Epidemiol. 14, 2004, 249-259, DOI:

10.1038/sj.jea.7500322.

Buratti F., Leoni C. and Testai E., The human metabolism of organophosphorothionate pesticides: consequences for toxicological risk assessment, J. Consum. Prot. Food Saf. 2, 2007, 37-44.

CDC, 2008. Laboratory Procedure Manual. Urinary Dialkylphosphate Metabolites. NHANES 20032004. Available from:

〈http://www.cdc.gov/nchs/data/nhanes/nhanes_03_04/I26opd_c_met_organophosphorus_pesticid es.pdf) (retrieved 18.12.13).

Curl C., et al., Organophosphorus pesticide exposure of urban and suburban preschool children with organic and conventional diets, Environ.Health Perspect. 111, 2003, 377-382 〈http://www.ncbi.nlm.nih.gov/pmc/articles/PMC1241395/〉.

Fenske R.A., et al., Strategies for assessing children's organophosphorus pesticide exposures in agricultural communities, J Expo Anal Environ Epidemiol 10, 2000, 662-671.

Field A., Discovering Statistics Using IBM SPSS Statistics, fourth ed., 2013, SAGE Publications Ltd.; London, England.

Fortin M.C., et al., Alteration of the expression of pesticide-metabolizing enzymes in pregnant mice: potential role in the increased vulnerability of the developing brain, Drug Metab. Dispos. 41, 2012, 326-331, DOI: 10.1124/dmd.112.049395. 
FSANZ, 2011. The 23rd Australian Total Diet Survey. Available from:〈http://www.foodstandards.gov.au/publications/pages/23rdaustraliantotald5367.aspx〉 (retrieved 18.12.13).

Health Canada. 2011. Results of the Canadian Health Measures Survey Cycle 1 (2007-2009). Report on Human Biomonitoring of Environmental Chemicals in Canada. Available from: 〈http://www.hcsc.gc.ca/ewh-semt/pubs/contaminants/chms-ecms/index-eng.php\#n5_8〉 (retrieved 26.12.13).

Heudorf U., et al., Current internal exposure to pesticides in children and adolescents in Germany: urinary levels of metabolites of pyrethroid and organophosphorus insecticides, Int. Arch. Occup. Environ. Health 77, 2004, 67-72.

Holland N., Furlong C., Bastaki M., Richter R., Bradman A., Huen K., Beckman K.and Eskenazi B., Paraoxonase polymorphisms, haplotypes, and enzyme activity in Latino mothers and newborns, Environ. Health Perspect. 114, 2006, 985-991.

Huen K., et al., Developmental changes in PON1 enzyme activity in young children and effects of PON1 polymorphisms, Environ. Health Perspect. 117, 2009, 1632-1638, DOI: 10.1289/ehp.0900870.

Kissel J.C., et al., Comparison of organophosphorus pesticide metabolite levels in single and multiple daily urine samples collected from preschool children in Washington State, J. Expo. Anal. Environ. Epidemiol. 15, 2005, 164-171, DOI: 10.1038/sj.jea.7500384.

Landrigan P. and Garg A., Children are not little adults, In: Pronzczuk de GarbinoJ., (Ed), Children's Health and the Environment: A Global Perspective - A Resource Manual for the Health Sector, 2005, World Health Organization; Geneva.

Lu C., et al., Dietary intake and its contribution to longitudinal organophosphorus pesticide exposure in urban/suburban children, Environ. Health Perspect. 116, 2008, 537-542, DOI: 10.1289/ehp.10912.

Lu C., et al., Organic diets significantly lower children's dietary exposure to organophosphorus pesticides, Environ. Health Perspect. 114, 2006, 260-263, DOI: 10.1289/ehp.8418.

Maclntosh D.L., et al., Longitudinal investigation of dietary exposure to selected pesticides, Environ. Health Perspect. 109, 2001, 145-150 〈http://www.ncbi.nlm.nih.gov/pmc/articles/PMC1240634//.

McGowan, R., 2003. Chemical Monitoring Survey of Victorian Certified Organic and Bio-dynamic Produce. Technical Report for Industry. Department of Primary Industries, Victoria. Retrieved from:〈http://www.dpi.vic.gov.au〉.

Monk, A., et al., 2012. Australian Organic Market Report 2012. Available from: 〈http://austorganic.com/wp-content/uploads/2013/09/Organic-market-report-2012-web.pdf) (retrieved 10.12.13).

Morgan M.K., et al., Exposures of preschool children to chlorpyrifos and its degradation product 3,5,6-trichloro-2-pyridinol in their everyday environments, J. Expo. Anal. Environ. Epidemiol. 15, 2005, 297-309, DOI: 10.1038/sj.jea.7500406.

Oates L., et al., Characteristics and consumption patterns of Australian organic consumers, J. Sci. Food Agric. 92, 2012, 2782-2787, DOI: 10.1002/jsfa.5664.

Oglobline A.N., et al., Negative ion chemical ionization GC/MS-MS analysis of dialkylphosphate metabolites of organophosphate pesticides in urine of non-occupationally exposed subjects, Analyst 126, 2001, 1037-1041, DOI: 10.1039/b102004h. 
Radcliffe, J.C., 2002. Pesticide Use in Australia. A Review Undertaken by the Australian Academy of Technological Sciences and Engineering, Melbourne. pp. 310.

Rauch S.A., et al., Associations of prenatal exposure to organophosphate pesticide metabolites with gestational age and birthweight, Environ. Health Perspect. 120, 2012, 1055-1060, (doi:10.1289\%2Fehp.1104615).

Riederer A.M., et al., Diet and nondiet predictors of urinary 3-phenoxybenzoic acid in NHANES 19992002, Environ. Health Perspect. 116, 2008, 1015-1022, DOI: 10.1289/ehp.11082.

Ross S.M., et al., Neurobehavioral problems following low-level exposure to organophosphate pesticides: a systematic and meta-analytic review, Crit. Rev. Toxicol. 43, 2013, 21-44, DOI: 10.3109/10408444.2012.738645.

Sudakin D.L. and Stone D.L., Dialkyl phosphates as biomarkers of organophosphates: the current divide between epidemiology and clinical toxicology, Clin. Toxicol. 49, 2011, 771-781, DOI: 10.3109/15563650.2011.624101.

Tasiopoulou S., et al., Results of the monitoring program of pesticide residues in organic food of plant origin in Lombardy (Italy), J. Environ. Sci.Health B 42, 2007, 835-841, DOI:

10.1080/03601230701555054.

The Expert Committee on Pesticide Residues in Food, 2013. Pesticide Residues Monitoring Programme for Quarter 1 2013. Available from:

〈http://www.pesticides.gov.uk/Resources/CRD/PRiF/Documents/Results\%20and\%20Reports/2013/2 013\%20Q1\%20Final.pdf $\rangle$ (retrieved 3 December).

United States Department of Agriculture, 2013. Pesticide Data Program: Annual Summary, Calendar Year 2011. Available from:

〈http://www.ams.usda.gov/AMSv1.0/getfile?dDocName=stelprdc5102692〉 (retrieved 03.10.13).

USDA, 2012. 2010-2011 Pilot Study Pesticide Residue Testing of Organic Produce. Available from: 〈http://www.ams.usda.gov/AMSv1.0/getfile?dDocName=STELPRDC5101234〉 (retrieved 15.12.13).

Willer, H., et al., 2013. The World of Organic Agriculture - Statistics and Emerging Trends 2013. Research Institute of Organic Agriculture (FiBL), Frick, and International Federation of Organic Agriculture Movements (IFOAM), Bonn.

Wilson N.K., et al., Aggregate exposures of nine preschool children to persistent organic pollutants at day care and at home, J. Expo. Anal. Environ. Epidemiol. 13, 2003, 187-202, DOI:

10.1038/sj.jea.7500270 